Open Access

\title{
Nuclear-enriched abundant transcript 1 as a diagnostic and prognostic biomarker in colorectal cancer
}

Yuchen $\mathrm{Wu}^{1+}$, Li Yang ${ }^{1 \dagger}$, Jiang Zhao ${ }^{1}$, Cong Li ${ }^{1}$, Jia Nie ${ }^{2}$, Fangqi Liu' ${ }^{1}$, Changhua Zhuo ${ }^{3}$, Yaxin Zheng ${ }^{4^{*}}$, Bin Li ${ }^{2^{*}}$, Zhimin Wang $^{5^{*}}$ and Ye Xu ${ }^{1 *}$

\begin{abstract}
Background: High expression of the long non-coding RNA nuclear-enriched abundant transcript 1 (NEAT1) in whole blood has been reported in colorectal cancer patients; however, its' clinical significance and origin are unclear. We evaluated the diagnostic and prognostic value, and origin of whole blood NEAT1 in colorectal cancer.

Methods: Expression of NEAT1 variants, NEAT1_v1 and NEAT1_v2 were determined using real-time quantitative PCR. The diagnostic value of whole blood NEAT1 expression was evaluated in test $(n=60)$ and validation $(n=200)$ cohorts of colorectal cancer patients and normal controls (NCS). To identify the origin of NEAT1, its expression was analyzed in blood, matched primary tumor tissues, para-tumor tissues, metastatic tissues, and also immune cells from patients or NCs. Function of NEAT1 in colorectal cell lines was also assessed. The correlation of NEAT1 expression with clinical outcomes was assessed in 191 patients.

Results: Whole blood NEAT1 expression was significantly higher in colorectal cancer patients than in NCs. NEAT1_v1 and NEAT1_V2 expression were highly accurate in distinguishing colorectal cancer patients from NCs (area under the curve: 0.787 and 0.871 , respectively). Knockdown of NEAT1_v1 in vitro could inhibit cell invasion and proliferation, while knockdown of NEAT1_v2 promoted cell growth. However, whole blood expression was not correlated with matched tissues. An elevated expression was seen in neutrophils from CRC patients. Furthermore, high expression of NEAT1_v1 was correlated with worse overall survival. In contrast, high expression of NEAT1_V2 alone was correlated with better overall survival.
\end{abstract}

Conclusion: Whole blood NEAT1 expression is a novel diagnostic and prognostic biomarker of overall survival in colorectal cancer. Elevated NEAT1 may derive from neutrophils.

Keywords: Biomarker, Prognosis, Diagnosis, Colorectal cancer, Long non-coding RNA

\footnotetext{
* Correspondence: zhengehbh@sina.com; binli@sibs.ac.cn; wangzhm@chgc.

sh.cn; xu_shirley021@163.com

${ }^{\dagger}$ Equal contributors

${ }^{4}$ Eastern Hepatobiliary Hospital, Second Military Medical University, No. 225

Chang-hai Road, Shanghai 200438, People's Republic of China

${ }^{2}$ Key Laboratory of Molecular Virology \& Immunology, Unit of Molecular

Immunology, Institut Pasteur of Shanghai, Shanghai Institutes for Biological Sciences, Chinese Academy of Sciences, No. 320 Yue-yang Road, Shanghai

20031, People's Republic of China

${ }^{5}$ Department of Genetics, Shanghai-MOST Key Laboratory of Health and

Disease Genomics, Chinese National Human Genome Center and Shanghai Industrial Technology Institute (SITI), No. 250 Bi-bo Road, Shanghai 201203, People's Republic of China

'Department of Colorectal Surgery, Fudan University Shanghai Cancer Center; Department of Oncology, Shanghai Medical College, Fudan University, No. 270 Dong-an Road, Shanghai 20032, People's Republic of China

Full list of author information is available at the end of the article
}

C Biomed Central (c) 2015 Wu et al. Open Access This article is distributed under the terms of the Creative Commons Attribution 4.0 International License (http://creativecommons.org/licenses/by/4.0/), which permits unrestricted use, distribution, and reproduction in any medium, provided you give appropriate credit to the original author(s) and the source, provide a link to the Creative Commons license, and indicate if changes were made. The Creative Commons Public Domain Dedication waiver (http://creativecommons.org/publicdomain/zero/1.0/) applies to the data made available in this article, unless otherwise stated. 


\section{Background}

Current evidence indicates that RNAs play important roles in oncogenesis and cancer pathogenesis. Considering that protein-coding sequences constitute only a small proportion of the genome, non-protein coding RNAs (ncRNAs), which constitute $>70 \%$ of the human genome [1], are becoming the subject of increasing attention because of their powerful regulator roles [2]. It is evident that ncRNA, like microRNAs and long ncRNAs (lncRNA) have roles in the emergence of a wide range of human diseases including cancers [3-5]. Commonly, lncRNAs are defined as ncRNAs longer than 200 nucleotides. They have multiple functions including acting as structural components, regulating protein trafficking, regulating transcription and cell metabolism, and modulating protein and RNA activity [6, 7].

Our former study using gene expression microarray revealed the non-protein coding RNAs (ncRNAs) - nuclearenriched Abundant Transcript 1 (NEAT1) elevated in peripheral blood from patients with CRC compared with healthy participants [8].

NEAT1 is an essential component of nuclear paraspeckles $[9,10]$. Paraspeckles are formed by the binding of Paraspeckle Protein (PSP) 1, PSP2 and p54 ${ }^{\text {nrb }}$ to the NEAT1 transcriptional start site $[11,12]$. Besides participating in mediate the development of corpus luteum and mammary gland $[13,14]$, nuclear paraspeckles have various functional significance. Especially, these nuclear bodies may serve as a "reservoir" for mRNA nuclear retention. They have also been shown to migrate to the cytoplasm to modulate cytoplasmic proteins and RNA function [12]. The NEAT1 gene encodes two transcripts: 3.7- kb NEAT1_v1 and 23- kb NEAT1_v2 [15, 16]. Although these two variants share the same transcriptional start site, NEAT1_v2 has a tRNA-like structure at its 3' end that is processed by RNaseP cleavage rather than a poly-A tail [15]. So the two variants shared two distinct 3 '-end processing mechanisms: canonical polyadenylation for NEAT1_v1 and RNase P-mediated cleavage for NEAT1_v2. NEAT1_v2 has been strongly implicated in the formation of paraspeckles [12, 15].

In the present study, we determined the diagnostic and prognostic significance and of whole blood NEAT1 in patients with colorectal cancer.

\section{Results}

Whole-blood NEAT1 expressions could distinguish patients with colorectal cancer from NCs

NEAT1_v1 and NEAT1_v2 levels were significantly higher in patients with colorectal cancer $(n=30)$ than in NCs $(n=30 ; p=0.0021$ and $p<0.0001$, respectively; Fig. 1a-b). Moreover, ROC analysis revealed that NEAT1 expression could distinguish patients with CRC from NCs (NEAT1_v1: area under the curve [AUC] $=0.732$, $95 \%$ confidence interval $[\mathrm{CI}]=0.602-0.838$; NEAT1_v2:
AUC $=0.845,95 \% \mathrm{CI}=0.728-0.925$; Fig. 1c-d). The sensitivity and specificity of NEAT1_v1 and NEAT1_v2 in identifying colorectal cancer were $56.7 \%$ and $86.6 \%$, $83.3 \%$ and $83.3 \%$, respectively.

The diagnostic value of NEAT1 was validated in a larger, independent cohort of colorectal cancer patients $(n=100)$ and NCs $(n=100)$. The expression of both NEAT1 variants was significantly higher in colorectal cancer patients than in NCs (both $p<0.0001$; Fig. 1e-f). ROC analysis confirmed the diagnostic potential of NEAT1 variants in the validation cohort. NEAT1_v1 yielded an AUC of 0.787 (95 \% CI = $0.724-0.842$; Fig. 1g) sensitivity of $69.0 \%$, and specificity of $79.0 \%$, and NEAT1_v2 yielded an AUC of 0.871 (95\% CI $=0.816-0.914$; Fig. $1 \mathrm{~h})$, sensitivity of $70.0 \%$, and specificity of $96.0 \%$. Interestingly, whole blood expression of NEAT1_v1, but not NEAT1_v2, was higher in patients with stage IV colorectal cancer compared with other stages $(p<0.0001$ and $p=0.132$, respectively; Fig. 1i-j).

\section{Functional assessment of NEAT1 in colorectal cancer cells}

We assessed cellular functions such as proliferation, and invasion after treatments with relevant siRNAs in two colorectal cancer cell lines. Knockdown of NEAT1_v1 could attenuated the invasive capacity, compared with cells transfected with NEAT1_v2 and control siRNA. Moreover, CCK8 assays revealed that down regulation of NEAT1_v1 lead to significant inhibition of cell growth. On the contrary, an elevated cell growth was detected when cells were treated with NEAT1_v2 siRNA (see Additional file 1).

\section{Validation of NEAT1 expression in tissues}

To further validate that whole blood NEAT1 levels might accurately reflected the concentration in colorectal cancer tissues, we determined the relationship between NEAT1 expression in primary colorectal cancer tissues, para-tumor tissues with matched whole blood.

Twenty-nine patients from validation cohort with stage I to stage IV colorectal cancer were enrolled. However, no correlation of both NEAT1 variants was found between tissues and blood (see Additional file 2).

Notably, we found a higher content of NEAT1_v1 in patients with metastasis colorectal cancer before. So we also inferred that such higher expression might derived from metastasis tissues rather than primary tumor tissues.

First of all, we examed another 19 patients who underwent simultaneously surgery for primary cancer and hepatic metastasis. Expression of NEAT1_v1 was significantly higher in metastatic hepatic tissues compared with para-tumor and primary colorectal cancer tissues $(p=0.003)$. NEAT1_v1 expression was similar between 


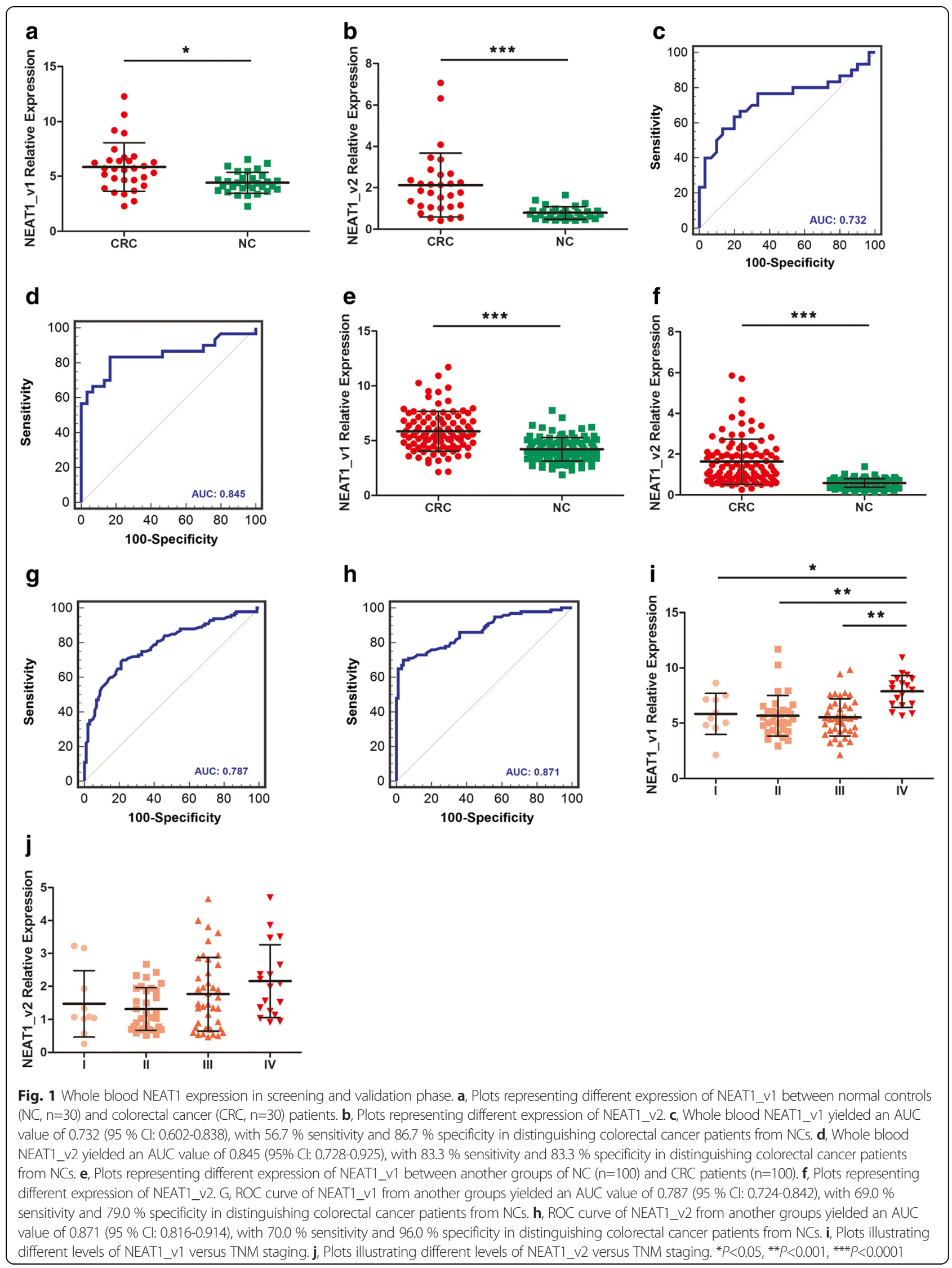


primary tumor and para-tumor tissues $(p=0.8214)$. NEAT1_v2 expression was not significantly different among para-tumor, primary tumor, and metastatic hepatic tissues $(p=0.076$; Fig. $2 \mathrm{a}-\mathrm{b})$. These results were somewhat in accordance with what we found in whole blood. However, after comparing the expression of both NEAT1 variants in whole blood and metastasis tissues, we also found no correlation between them (see Additional file 3), which meant that there might be other reasons for such difference in whole blood rather than tissue origins.

\section{Neutrophil expression of NEAT1 variants was higher in colorectal cancer patients than in NCs}

There was no correlation of NEAT1 expression between whole blood and tissues. Considering that immune cells comprise the majority of peripheral blood cells, we also determined NEAT1 expression from different subgroups of immune cells between colorectal cancer patients and NCs.

We separated neutrophils, monocytes, CD4-positive cells (mainly CD4-positive $\mathrm{T}$ helper cells), CD8-positive cells (mainly cytotoxic T cells), and CD4/CD8 doublenegative cells (mainly B cells and natural killer cells) from peripheral blood (see Additional file 4).

Neutrophils had the highest expression of both NEAT1 variants among the immune cell types. Expression of both variants in neutrophils were higher in colorectal cancer patients than in NCs (NEAT1_v1, $p=0.001$; NEAT1_v2, $p=0.015$; Fig. $3 \mathrm{a}-\mathrm{b})$. Moreover, when comparing neutrophils' expression in different cancer stages, an elevated content of NEAT1_v1 but not NEAT1_v2 was found in stage IV patients, which was in accordance with what we found in whole blood (NEAT1_v1 Stage IV, $p<0.001$,
Fig. 3c-d). Overall, we testified that NEAT1 expression in peripheral neutrophils might be the key point in distinguishing CRC patients from healthy people.

\section{Whole blood expression of NEAT1 was significantly correlated with overall survival in patients with colorectal cancer}

After proving the diagnostic value of whole blood expression of NEAT1, we proceeded to verify its function in prognosis. We determined the correlation of whole blood NEAT1_v1 and NEAT1_v2 expression with clinical outcomes in 191 colorectal cancer patients with available clinicopathological data. NEAT1_v1 were significantly higher in colorectal cancer patients with distant metastases (M1) than in those without metastasis (M0; $p=0.023)$, whereas NEAT1_v2 levels did not differ between the M1 and M0 groups ( $p=0.9917$; Table 1 and Fig. 4a).

The median follow-up duration was 56 (2-83) months. Patients with high NEAT1_v1 expression had shorter OS and median survival times than patients with low NEAT1_v1 expression ( $p=0.003$; Fig. 4b).

Univariate analysis revealed that nodal status (N0/N1 $p<0.001$; N0/N2 $p=0.001)$, distant metastasis $(p<0.001)$, lymphatic/vascular invasion $(p<0.001)$, perineural invasion $(p=0.001)$, extranodal tumor deposit $(p=0.005)$, American Joint Committee on Cancer stage $(p<0.001)$, and NEAT1_v1 expression ( $p=0.004)$ were significantly associated with OS. However, in the multivariate analysis, only nodal status (N0/N1 $p<0.001 ; \mathrm{N} 0 / \mathrm{N} 2 p=0.017)$ and distant metastasis $(p<0.001)$ remained significantly independent prognostic factors for OS (Table 2). NEAT1_v1 expression was found to have limited prognostic value for OS $(p=0.068)$.
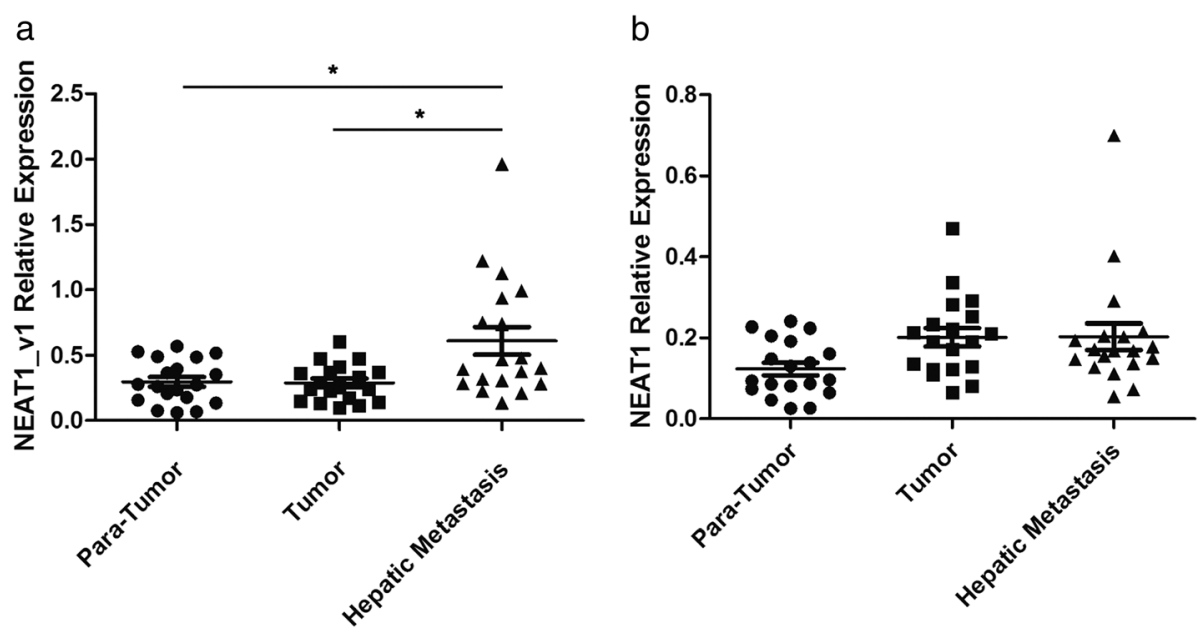

Fig. 2 NEAT1 expression differed in the para-tumor, tumor and matched liver metastasis. (a) Significantly higher expression of NEAT1_v1 was detected in liver metastasis samples versus para-tumor tissues and tumor tissues $(p=0.003)$. (b) No significant difference was detected among primary tumor tissues, para-tumor tissues and liver metastasis ( $p=0.076)$. ${ }^{*} A$ two-tailed $p$ value $\leq 0.05$ was considered statistically significant 

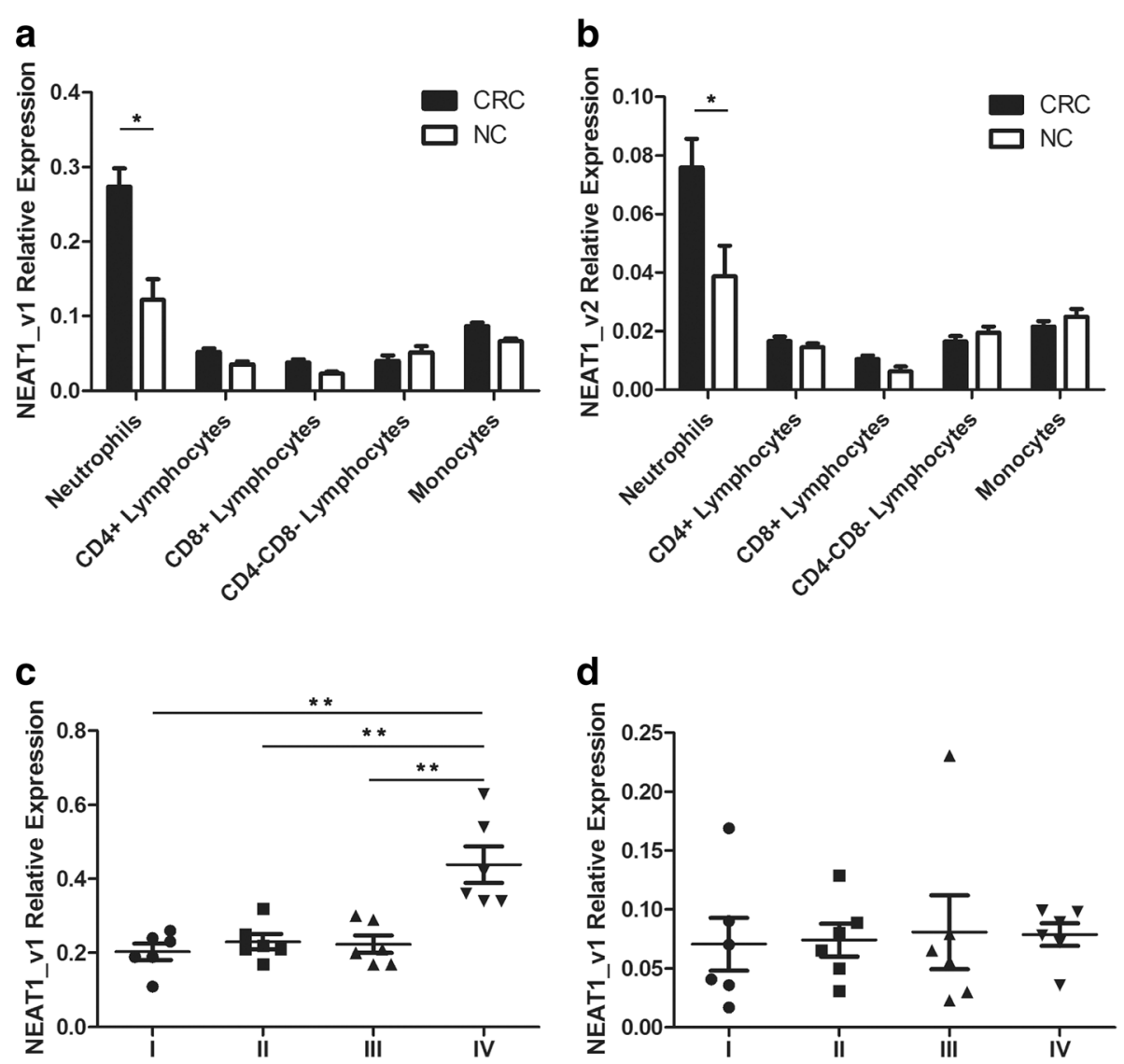

Fig. 3 Different expression of NEAT1 isoforms in 5 groups of immune cells sorted from peripheral blood. (a) The NEAT1_v1 expression was different in five groups of immune cells, and varied dramatically in neutrophils from two groups of people $(p=0.018)$. (b) The NEAT1_V2 expression in CRC patients was also higher than that in normal controls $(p=0.0057)$. All graphs showed mean values \pm SEM. ${ }^{*} p<0.05$. (c) NEAT1_v1 was higher in neutrophils fro stage IV colorectal cancer patients. (d) No significant difference was detected in NEAT1_V2 expression in different stage

Although OS did not differ in patients with different levels of NEAT1_v2 ( $p=0.281$, Fig. 4c), higher expression of NEAT1_v2 without higher expression of NEAT1_v1 (high expression of NEAT1_v2 alone) represented better OS compared with low and high expression of both variants ( $p=0.036$ and $p<0.001$, respectively) (Fig. 4d). There was no significant difference in OS between patients with low expression of both variants compared with those with high expression of both variants $(p=0.303)$. Multivariate analysis, revealed that high expression of NEAT1_v2 alone could be an independent prognostic factor for improved OS $(p=0.0038)$. Moreover, we found that higher expression of NEAT1_v1 was almost combined with higher expression of NEAT1_v2 (107/111 patients).

\section{Discussion}

Colorectal cancer is one of the most common malignancies worldwide. It is the third common cancer and the fifth leading cause of death in China [17, 18]. Early diagnosis of colorectal cancer is important for improving treatment response, patients' survival, and quality of life. Blood-based tests to detect tumor markers, such as carcinoembryonic antigen, may help to evaluate disease status, but their accuracy and efficacy are controversial $[19,20]$. Ideally, diagnostic tests for the early detection of colorectal cancer should be simple, accurate, and minimally invasive. Considerable studies have identified and developed novel biomarkers for diagnosis, prognosis, and predicting treatment response [21-24]. Using independent patient cohorts, we demonstrated for the first time that whole blood NEAT1_v1 and NEAT1_v2 may be valuable diagnostic biomarkers in colorectal cancer. However, NEAT1_v2 seemed to be a more sensitive and specific biomarker compared with NEAT1_v1 (70 \% vs. $69.0 \%$ and $96.0 \%$ vs. $79.0 \%$, respectively).

Our study also demonstrated the prognostic potential of NEAT1 expression in colorectal cancer. Whole blood NEAT1_v1 expression was higher in patients with distant metastasis, and patients with high NEAT1_v1 expression 
Table 1 Relationship between NEAT1 and clinical features of patients with colon cancer in the whole blood

\begin{tabular}{|c|c|c|c|c|c|c|}
\hline \multirow[t]{2}{*}{ Clinical variables } & \multirow[t]{2}{*}{ Classification } & \multirow[t]{2}{*}{$N$} & \multicolumn{2}{|c|}{ NEAT1_v1 } & \multicolumn{2}{|c|}{ NEAT1_v2 } \\
\hline & & & Mean & $P$ value & Mean & $P$ value \\
\hline \multirow[t]{2}{*}{ Gender } & Male & 101 & 6.26 & 0.098 & 1.65 & 0.987 \\
\hline & Female & 90 & 5.38 & & 1.65 & \\
\hline \multirow[t]{2}{*}{ Age (years) ${ }^{a}$} & $<56$ & 100 & 5.60 & 0.253 & 1.62 & 0.769 \\
\hline & $\geq 56$ & 91 & 6.21 & & 1.69 & \\
\hline \multirow[t]{2}{*}{ Tumor (T) stage } & $\mathrm{T} 1-2$ & 26 & 6.16 & 0.287 & 1.46 & 0.476 \\
\hline & T3-4 & 165 & 5.80 & & 1.68 & \\
\hline \multirow[t]{3}{*}{ Nodal (N) status } & No & 95 & 5.45 & 0.287 & 1.54 & 0.464 \\
\hline & N1 & 48 & 6.02 & & 1.65 & \\
\hline & N2 & 48 & 6.45 & & 1.87 & \\
\hline \multirow[t]{2}{*}{ Distant metastasis (M) } & MO & 170 & 5.52 & $0.023^{*}$ & 1.63 & 0.532 \\
\hline & M1 & 21 & 8.49 & & 1.84 & \\
\hline \multirow[t]{2}{*}{ AJCC stage $^{\mathrm{b}}$} & $|-| \mid$ & 94 & 5.45 & 0.144 & 1.55 & 0.374 \\
\hline & III-IV & 97 & 6.23 & & 1.74 & \\
\hline \multirow[t]{2}{*}{ Lymphovascular invasion } & No & 141 & 5.55 & 0.497 & 1.70 & 0.463 \\
\hline & Yes & 50 & 6.67 & & 1.52 & \\
\hline \multirow[t]{2}{*}{ Perineural invasion } & No & 163 & 5.55 & 0.549 & 1.67 & 0.701 \\
\hline & Yes & 28 & 7.54 & & 1.55 & \\
\hline \multirow[t]{2}{*}{ Extranodal tumor deposits } & No & 163 & 5.53 & 0.139 & 1.66 & 0.800 \\
\hline & Yes & 28 & 7.66 & & 1.58 & \\
\hline \multirow[t]{2}{*}{ Differentiation } & G1-G2 & 149 & 5.92 & 0.587 & 1.64 & 0.880 \\
\hline & G3-G4 & 42 & 5.57 & & 1.68 & \\
\hline \multirow[t]{2}{*}{ Pathology } & Adenocarcinoma & 164 & 5.93 & 0.412 & 1.68 & 0.465 \\
\hline & Mucinous or signet-ring carcinoma & 27 & 5.30 & & 1.46 & \\
\hline \multirow[t]{2}{*}{ Maximum size $(\mathrm{cm})^{\mathrm{a}}$} & $<5$ & 118 & 6.02 & 0.344 & 1.67 & 0.687 \\
\hline & $>5$ & 73 & 5.48 & & 1.58 & \\
\hline
\end{tabular}

${ }^{*}$ A two-tailed $p$ value $\leq 0.05$ was considered statistically significant

${ }^{\mathrm{a}}$ The mean age and tumor size, respectively

${ }^{b}$ Abbreviations: AJCC, American Joint Committee on Cancer (AJCC)

had poorer OS. However, NEAT1_v1 expression was found not to be an independent prognostic factor for OS in the multivariate analysis. Furthermore, NEAT1_v1 was higher in hepatic metastatic tissues than in primary colorectal cancer tissues, suggesting that elevated NEAT1_v1 expression in whole blood may originate from metastatic tissues. Further studies are needed to assess the potential contribution of other metastatic sites such as the lung and bone to NEAT1_v1 expression in the peripheral whole blood of colorectal cancer patients.

Interestingly, despite sharing the same transcriptional start site, NEAT1_v1 and NEAT1_v2 appeared to have different roles in predicting clinical outcomes. We found that high NEAT1_v2 combined with low NEAT1_v1 expression was associated with improved OS in colorectal cancer patients. Furthermore, NEAT1_v2 expression was an independent prognostic factor for OS in the multivariate analysis. In contrast, elevated NEAT1_v1 was associated with poor OS because of its association with metastasis. We also found that patients with simultaneous low or high expression of NEAT1_v1 and NEAT1_v2 had poor OS. Based on these findings, we believe that NEAT1_v2 may play a protective role in colorectal cancer. In patients with low expression of both variants, NEAT1_v2 is unable to perform its protective function. On the other hand, in patients with high expression of both variants, the putative protective role of NEAT1_v2 may be masked by high NEAT1_v1 expression, resulting in poor OS and tendency toward metastasis. Although mechanisms underlying this dynamic equilibrium may be difficult to investigate, it is clear that the differential expression of NEAT1 variants in whole blood has prognostic value in colorectal cancer.

The ratio of the two variants of NEAT1 is regulated by differential use of polyadenylation signals. Naganuma et al. demonstrated that one of the paraspeckle proteins 


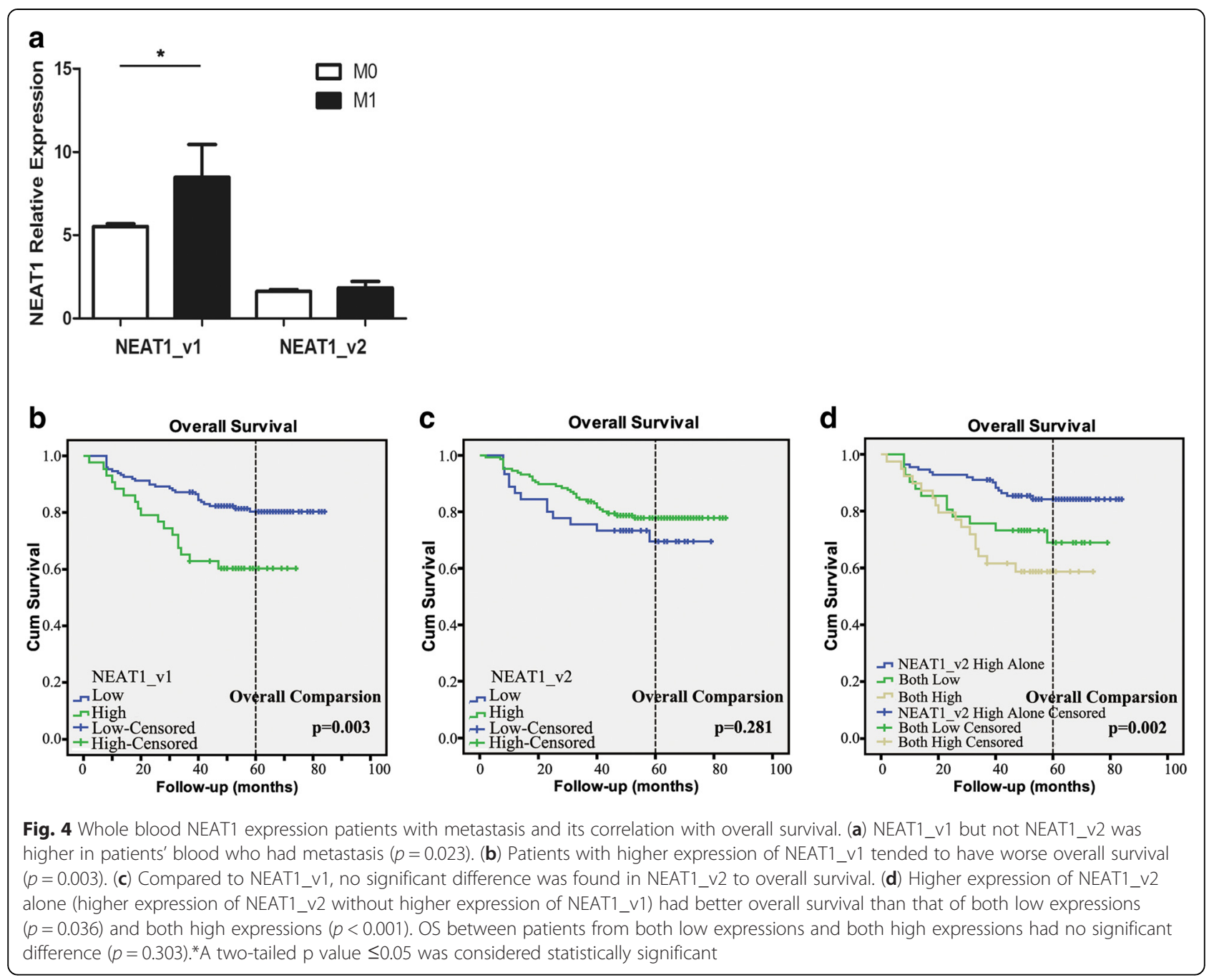

(PSPs), HNRNPK competed with CPSF6 (CFIm68) for binding to NUDT21 (CFIm25), leading to the accumulation of NEAT1_v2 [25]. Interestingly, Chioniso et al. indenified CFIm25 as a tumour suppresser as RNA 3'end-processing factor in glioblastoma [26]. So further research on the polyadenylation signals in NEAT1 edition and its function in colorectal cancer is required. Furthermore, other roles of NEAT1 in mediating tumorigenesis and metastasis has recently received increasing attention [27-29]. Choudhry et al. found that NEAT1 was regulated by hypoxia-inducible factor- 2 and hypoxia-induced NEAT1 accelerates cellular proliferation, improves cell survival, and reduces apoptosis (28). Chakravarty et al. reported that androgen receptor antagonist-resistant prostate cancer cells express high levels of NEAT1, which was shown to drive their oncogenic growth (29). In the present study, we demonstrated that NEAT1_v1 could improve tumor cells growth and invasion, while NEAT1_v2 had reverse function in proliferation, which was somewhat in corresponding with the prognostic potential of whole blood NEAT1 expression. Moreover, we found that NEAT1_v1 was highly expressed in metastatic hepatic tissues from colorectal cancer patients. These findings suggest that NEAT1 has both pro-oncogenic and pro-metastatic effects in colorectal cancer. A recently study demonstrated that NEAT1 and another lncRNA, metastasis-associated lung adenocarcinoma transcript 1 (MALAT1) colocalize to hundreds of genomic sites and may have complementary functions [30]. MALAT1, an 8.1 $\mathrm{kb}$ lncRNA , is overexpressed in many malignant tumors, including human primary colorectal cancer. MALAT1 upregulation has been reported to promote colorectal cancer development via its target protein A-kinase anchor protein [31]. Together, these studies indicate that NEAT1 has a potentially complex role in driving tumorigenesis.

In the present study, we also investigated the possible sources contributing to the differential whole blood expression of NEAT1 between colorectal cancer patients 
Table 2 Cox analysis of the prognostic variables on the overall survival in patients (Whole blood)

\begin{tabular}{|c|c|c|}
\hline \multirow[t]{2}{*}{ Prognosis variables } & \multicolumn{2}{|c|}{ Overall survival } \\
\hline & $P$ value & $\mathrm{HR}(95 \% \mathrm{Cl})$ \\
\hline \multicolumn{3}{|l|}{ Univariate analysis } \\
\hline NEAT1_v1, Low/High & $0.004^{*}$ & $0.415(0.227-0.758)$ \\
\hline NEAT1_v2, Low/High & 0.285 & $1.421(0.746-2.708)$ \\
\hline NEAT1_v2, High Alone ${ }^{a}$ & $0.006^{*}$ & $0.426(0.231-0.784)$ \\
\hline Gender, Male/Female & 0.399 & $1.290(0.714-2.330)$ \\
\hline Age, $<56 / \geq 56$ years $^{b}$ & 0.967 & $0.988(0.544-1.793)$ \\
\hline Tumor (T) stage, $\mathrm{T} 1-2 / \mathrm{T} 3-4$ & 0.124 & $0.399(0.124-1.288)$ \\
\hline Nodal (N) status, N0/N1 & $<0.001^{*}$ & $0.120(0.056-0.257)$ \\
\hline N0/N2 & $<0.001^{*}$ & $0.297(0.143-0.617)$ \\
\hline Distant metastasis (M), M0/M1 & $<0.001^{*}$ & $0.102(0.055-0.188)$ \\
\hline Lymphatic/vascular invasion, No/Yes & $<0.001^{*}$ & $0.243(0.135-0.438)$ \\
\hline Perineural invasion, No/Yes & $0.001^{*}$ & $0.342(0.179-0.653)$ \\
\hline Extranodal tumor deposit, No/Yes & $0.005^{*}$ & $0.389(0.201-0.753)$ \\
\hline AJCC Stage ${ }^{c},|-||/|||-\mid V$ & $<0.001^{*}$ & $0.205(0.066-0.426)$ \\
\hline Differentiation, G1-G2/G3-G4 & 0.058 & $0.543(0.289-1.022)$ \\
\hline Pathology, Adenocarcinoma/Mucinous or signet-ring carcinoma & 0.712 & $0.859(0.384-1.923)$ \\
\hline Size, $<5 / \geq 5^{b}$ & 0.598 & $1.187(0.627-2.247)$ \\
\hline \multicolumn{3}{|l|}{ Multivariate analysis } \\
\hline NEAT1_v1, Low/High & 0.068 & \\
\hline NEAT1_v2, High Alone ${ }^{a}$ & $0.038^{*}$ & $0.519(0.280-0.965)$ \\
\hline Nodal (N) status, N0/N1 & $<0.001^{*}$ & $0.199(0.088-0.452)$ \\
\hline N0/N2 & $0.017^{*}$ & $0.402(0.189-0.851)$ \\
\hline Distant metastasis (M), M0/M1 & $<0.001^{*}$ & $0.193(0.099-0.374)$ \\
\hline Lymphatic/vascular invasion, No/Yes & 0.996 & \\
\hline Perineural invasion, No/Yes & 0.366 & \\
\hline Extranodal tumor deposit, No/Yes & 0.645 & \\
\hline AJCC Stage ${ }^{c},|-||/|||-\mid V$ & 0.498 & \\
\hline
\end{tabular}

${ }^{*}$ A two-tailed $p$ value $\leq 0.05$ was considered statistically significant

${ }^{\text {a }}$ High expression of NEAT1_v2 with low expression of NEAT1_v1

${ }^{\mathrm{b}}$ The mean age and tumor size, respectively

'Abbreviations: AJCC, American Joint Committee on Cancer (AJCC)

and NCs. The expression of NEAT1 was higher in patients' blood than tissues. We detected higher expression in metastasis liver tissues and proved its mechanism in tumor cells proliferation and invasion. The in vitro experiments enclosed a contradictory mechanism of NEAT1 variants in tumor development and invasion, which was in accord with the prognostic potential in whole blood. NEAT1_v1 acted as a carcinogenic factor, while NEAT1_v2 was a protective one. However, fact that no correlation was found between whole blood and tissues could not be ignored. One of the possible explanation might be the degradation of NEAT1 in whole blood which derived from tissues. Moreover, expression of NEAT1 was higher in whole blood than in tissues. This suggests that cancer-related sources do not fully account for the increased expression in whole blood. Our findings indicate that neutrophils may mostly contribute to the elevated expression of NEAT1 in whole blood, as both NEAT1_v1 and NEAT1_v2 expression were higher in neutrophils from colorectal cancer patients than in those from NCs, and a higher NEAT1_v1 expression was also detected in neutrophils from stage IV patients, which was in correspondence with the difference found in whole blood.

The functional roles of peripheral blood and tumorassociated neutrophils have been investigated in cancer patients. Clinical research has primarily evaluated the prognostic role of peripheral neutrophils in cancer patients [32-34]. These studies have shown that a high 
neutrophil to lymphocyte ratio is an independent prognostic factor for survival. On the other hand, fundamental research has mostly focused on tumorassociated neutrophils [35] and their migration [36-38], proliferation, correlation with other immune cells such as myeloid-derived suppressor cells [39-41], and paradoxical functions on tumorgenesis [42-45]. Therefore, the functional roles of peripheral neutrophils in tumorigenesis are still unclear. Although the role of NEAT1 in immune system function, especially during viral infection (e.g., human immunodeficiency viral infection), has been intensely investigated [46-48], its function of NEAT1 in neutrophils has not been fully addressed. Considering that NEAT1 acts as one of the main structural components in paraspeckles and possess a wide range of known or unknown function, its role in peripheral neutrophils of colorectal cancer patients is warranted. Whether increased NEAT1 expression in neutrophils is a cause or by-product of tumorigenesis and whether there are other potential sources of NEAT1 are still unclear.

Our findings indicated the significant promise of whole blood NEAT1 expression as a diagnostic marker in colorectal cancer and thus, warrants confirmation in larger studies. These studies should include patients in the same general condition and use a uniform method to detect RNA expression to help eliminate any potential coincidental bias. Differential expression of NEAT1 variants may be potentially relevant in other cancer types; therefore, further studies in patients with other cancer types are also needed. Moreover, despite no correlation of NEAT1 expression between whole blood and tissues was found, an elevated expression in metastasis was certainty. So basic research on NEAT1 in carcinogenesis is indispensable and is in process. Moreover, higher NEAT1 expression in CRC patients' whole blood did not correlated with tissues and it might originated from its different content in neutrophils. In summary, we believe that such difference in whole blood derived from numerous sources, but mainly from neutrophils. However, the function of NEAT1 on neutrophils needs to be uncovered in the future.

In conclusion, our study established and validated NEAT1 as a new diagnostic biomarker in colorectal cancer. In addition, NEAT1_v1 and NEAT1_v2 expression were associated with different prognostic outcomes. Elevated whole blood NEAT1 expression may derive, in part, from metastatic tissues and neutrophils. Further research is needed to fully clarify the clinical significance of high neutrophil NEAT1 expression in colorectal cancer patients.

\section{Materials and Methods} Study design and patients

The study design and patient characteristics are confirmed (see Additional files 5 and 6). Study participants were recruited from the Fudan University Shanghai Cancer Center (China) between 2006 and 2012. Patients who underwent any preoperative radiotherapy or chemotherapy before blood collection, and those with inflammatory bowel disease, hereditary colorectal cancer, or other rare tumor types were excluded. Healthy volunteers with normal colonoscopy results for colorectal cancer served as the normal controls (NCs). The study was approved by the Medical Ethics Committee of Fudan University Shanghai Cancer Center, and written informed consent was obtained from all participants. TNM classification and differentiation grade were determined according to the 2010 American Joint Committee on Cancer criteria (7th edition). Clinicopathological data were obtained retrospectively from the patients' electronic medical records.

The diagnostic value of whole blood NEAT1 was initially evaluated in a test cohort comprised of 30 colorectal cancer patients and $30 \mathrm{NCs}$ and then validated in a large, independent cohort of 100 patients and 100 NCs. Tumor tissues, para-tumor tissues of 29 patients (stage I to stage IV) from validation cohort were collected and compared with matched blood sample. Moreover, another 19 patients receiving simultaneously resection of primary tumor tissues and liver metastasis were also enrolled to investigate the correlation of NEAT1 between whole blood and tissues.

Subsequently, a survival analysis was performed in an additional 191 patients with available clinicopathological and prognostic data. Finally, to identify the origin of whole blood NEAT1 expression, NEAT1 expression in primary colorectal cancer and matched hepatic metastatic tissues and immune cells was determined. Primary colorectal cancer and matched hepatic metastatic tissues were obtained from 19 colorectal cancer patients who had undergone simultaneous resection of colorectal cancer and hepatic metastasis. Immune cells were isolated from peripheral blood samples obtained from 12 colorectal cancer patients and $12 \mathrm{NCs}$.

\section{Blood and tissue collection and immune cell isolation}

All blood samples were obtained prior to surgery or adjuvant therapy. To investigate NEAT1 expression for clinical outcomes, peripheral blood $(2.5 \mathrm{~mL})$ samples were collected into PAXgene Blood RNA Tubes (PreAnalytiX). Normal and cancerous colorectal tissues and matched hepatic metastatic tissues were collected immediately after surgery, washed in cold PBS, and stored in RNAlater RNA Stabilization Reagent (Qiagen) at $-20{ }^{\circ} \mathrm{C}$ for future use. For immune cell sorting, peripheral blood samples (20 $\mathrm{mL}$ ) were collected and stored in EDTA anticoagulant tubes. All immune cells were isolated $<12 \mathrm{~h}$ after collection. Neutrophils were isolated using improved Ficoll Solution (TBD). CD4-positive and CD8-positive T cells 
were isolated from peripheral blood mononuclear cells by fluorescence-activated cell sorting (Aria II Cell Sorter; BD Biosciences) with fluorescein isothiocyanate-conjugated anti-CD4 and allophyocyanin-conjugated anti-CD8 monoclonal antibodies (BD Biosciences) according to manufacturer's instructions. All fluorescence-activated cell sorting data were analyzed using FlowJo software (Tree Star).

\section{RNA isolation and quantitative real-time reverse transcription-PCR}

Total RNA was extracted from peripheral blood samples using the PAXgene Blood RNA System (PreAnalytiX). Total RNA was isolated from immune cells and tissues using TRIzol Reagent (Sigma) according to the manufacturer's instructions. RNA was quantified using a NanoDrop spectrophotometer. For each sample, RNA (320 ng) was reverse transcribed using the QuantiTect Reverse Transcription Kit (Qiagen). cDNA was amplified using SYBR Premix DimerEraser (Perfect Real Time; Takara Biotechnology), and real-time quantification was performed using the Applied Biosystems 7900HT Fast Real-Time PCR System (Life Technologies). As we had previously identified DECR1 (2,4-dienoyl-coenzyme A reductase 1 mitochondrial) as a stably expressed reference gene in whole blood samples $[8,49,50]$, it was used as a reference gene for the blood sample analysis. $\beta$-actin was used as a reference gene for the tissue sample analysis. Primer sequences are provided in Additional file 7. All realtime PCR products were verified by DNA sequencing. Relative gene expression levels were estimated using the comparative $\mathrm{Ct}$ method of relative quantification, normalizing the $\mathrm{Ct}$ values relative to the reference gene. Relative gene expression is presented as $2^{-\Delta C t}$, where $\Delta \mathrm{Ct}=\mathrm{Ct}$ colorectal cancer $-\mathrm{Ct}$ Control.

\section{Cell lines and cell culture conditions}

Two human colorectal cancer cell lines (HCT116, LOVO) were obtained from the Type Culture Collection of the Chinese Academy of Sciences (Shanghai, China) and were cultured in McCoy's 5a and F-12 K Medium respectively. All medium contained $10 \%$ fetal bovine serum (FBS). Medium and FBS were obtained from GIBCO $^{\circ}$ (Life Technologies, USA).

\section{Invasion and proliferation assays}

NEAT1 is located in cell nucleus and is hard to knockdown with simple siRNA stably. So we used a mixed siRNA pool to silence NEAT1 variants respectively. In transwell assay, transfected cells $\left(3 \times 10^{5}\right.$ cells/well $)$ were seeded in serum-free medium in 12-well Transwell Chamber (BD Biosciences, USA). 50ul diluted Matrigel was plated in chambers. They were placed in cell culture plates containing $10 \% \mathrm{FBS}$ and incubated for $48 \mathrm{~h}$. After that, membranes were washed, fixed and stained with
Crystal Violet. Invading cells in 10 microscopic fields were counted.

Cell Counting Kit 8 (CCK-8, Donjindo) was used to assessed cell viability. Treated cells were seeded into 96well plates at an initial density of $1 \times 10^{3}$ cells/well. After $24,48,72$, and $96 \mathrm{~h}$ of cultivation, CCK-8 solution was added to each well and incubated for $2 \mathrm{~h}$. The absorbance was measured by scanning with a microplate reader (MRX; Dynex Technologies, West Sussex, United Kingdom) at $450 \mathrm{~nm}$.

Each independent experiment was performed 3 times. Results are presented as mean $\pm \mathrm{SE}$.

\section{Statistical analyses}

Statistical analyses were performed using SPSS v.19.0 (IBM Corp.). Receiver operating characteristic (ROC) analysis was performed to determine the ability of whole blood NEAT1 expression to distinguish colorectal cancer patients from NCs. The Kolmogorov-Smirnov test was used to verify the normal distribution of cardinal variables. Exploratory comparison of normally distributed and non-normally distributed independent groups was performed using t-tests and Mann-Whitney U (2 groups) or Kruskal-Wallis tests ( $>2$ groups), respectively. The Friedman test was used to analyze differences among 3 paired samples. Pearson analysis was performed to explore the correlation of NEAT1 between blood and tissues. Overall survival (OS) was estimated using Kaplan-Meier curves and compared using the log-rank test. OS was calculated as the time from date of definitive diagnosis to date of death by any cause. Prognostic factors were determined using Cox regression analysis. A two-tailed $p$ value $<0.05$ was considered statistically significant.

\section{Ethics, consent and permissions}

Written informed consent was obtained from all participants. Study was approved by the Medical Ethics Committee of Fudan University Shanghai Cancer Center (reference number 050432-4-1212B).

\section{Consent to publish}

We have obtained consent from all participants to report data to publish.

\section{Additional files}

Additional file 1: Figure S1. Functional assays of NEAT1 on colorectal cancer cells. Knockdown of NEAT1_v1 expression suppressed cell invasion and proliferation, while knockdown of NEAT1_v2 promote cell proliferation. (PDF 395 kb)

Additional file 2: Table S1. Correlation of NEAT1 expression between blood and matched tissues. Blood and matched tissues were collected from 46 patients (Stage I-IV). Correlation analysis was performed and $p$-value was present in the table. (PDF 303 kb) 
Additional file 3: Table S2. Correlation of NEAT1 expression between blood and matched tissues in patients with simultaneously surgery for primary cancer and hepatic metastasis. Blood and matched tissues were collected from 19 patients with simultaneously surgery. Correlation analysis was performed and $p$-value was present in the table. (PDF $151 \mathrm{~kb}$ )

Additional file 4: Figure S2. 5 groups of immune cells separated from peripheral blood from patients and healthy donors. Neutrophils were isolated using improved Ficoll Solution. Monocytes, $\mathrm{CD}^{+}{ }^{+}$Lymphocytes, $\mathrm{CD}^{+}{ }^{+}$Lymphocytes, and $\mathrm{CD}_{4}^{-} \mathrm{CD} 8^{-}$Lymphocytes were isolated from peripheral blood mononuclear cells by fluorescence-activated cell sorting with anti-CD4 and anti-CD8 monoclonal antibodies. (PDF $85.8 \mathrm{~kb}$ )

Additional file 5: Figure S3. Study design. The blood gene expression was identified using a screening set and an independent validation set. Blood from another 191 patients was detected for clinical outcomes. Primary lesion and metastasis with matched blood were also investigated. Another group of blood were detected on immune cells. (PDF $87.5 \mathrm{~kb}$ )

Additional file 6: Table S3. Characteristics of the CRC and control populations. (PDF $183 \mathrm{~kb}$ )

Additional file 7: Table S4. Primer sequences for NEAT1 and reference genes. (PDF $132 \mathrm{~kb}$ )

\section{Abbreviations}

NEAT1: Nuclear-enriched abundant transcript 1; CRC: Colorectal cancer; PSP: Paraspeckle protein; IncRNA: Long non-coding RNA; ROC: Receiver operating characteristic curve; AUC: Area under the curve; OS: Overall survival; NC: Normal control; AJCC: American joint committee on cancer; TAN: Tumor associated neutrophil; DECR1: 2,4-dienoyl-coenzyme A reductase 1 mitochondrial; MALAT1: Metastasis-associated lung adenocarcinoma transcript 1.

\section{Competing interests}

The authors have no conflict of interest to disclose.

\section{Authors' contributions}

YCW, YX, ZMW, BL and YXZ conceived and designed the study. JZ and CL collected the clinical samples and data. YCW and LY carried out the most experiments, YCW and JN participated in the FACS sorting. YCW, CHZ, FQL and ZMW analyzed the data. YCW drafted the manuscript. YX, ZMW, YXZ and $B L$ revised the manuscript for important intellectual content. All authors read and approved the final manuscript.

\section{Acknowledgements}

This work was partially supported by the National Natural Science Foundation of China (grant nos. 81472620, 81272307), and Pudong New Area Science and Technology Development Fund (Pkj2013-z02). The author thanks Xun Ye for his assistance with data collection.

\section{Author details}

${ }^{1}$ Department of Colorectal Surgery, Fudan University Shanghai Cancer Center; Department of Oncology, Shanghai Medical College, Fudan University, No. 270 Dong-an Road, Shanghai 20032, People's Republic of China. ${ }^{2}$ Key Laboratory of Molecular Virology \& Immunology, Unit of Molecular Immunology, Institut Pasteur of Shanghai, Shanghai Institutes for Biological Sciences, Chinese Academy of Sciences, No. 320 Yue-yang Road, Shanghai 20031, People's Republic of China. ${ }^{3}$ Department of Surgical Oncology, Fujian Provincial Cancer Hospital, Teaching Hospital of Fujian Medical University, No. 420 Fu-ma Road, Fuzhou 350014, People's Republic of China. Eastern Hepatobiliary Hospital, Second Military Medical University, No. 225 Chang-hai Road, Shanghai 200438, People's Republic of China. ${ }^{5}$ Department of Genetics, Shanghai-MOST Key Laboratory of Health and Disease Genomics, Chinese National Human Genome Center and Shanghai Industrial Technology Institute (SITI), No. 250 Bi-bo Road, Shanghai 201203, People's Republic of China.

Received: 13 April 2015 Accepted: 13 October 2015

Published online: 09 November 2015

\section{References}

1. Carninci P, Kasukawa T, Katayama S, Gough J, Frith MC, Maeda N, et al. The transcriptional landscape of the mammalian genome. Science. 2005;309(5740):1559-63.

2. Wilusz JE, Sunwoo H, Spector DL. Long noncoding RNAs: functional surprises from the RNA world. Genes Dev. 2009;23(13):1494-504.

3. Mercer TR, Dinger ME, Mattick JS. Long non-coding RNAs: insights into functions. Nat Rev Genet. 2009;10(3):155-9.

4. Zhang H, Chen Z, Wang X, Huang Z, He Z, Chen Y. Long non-coding RNA: a new player in cancer. J Hematol Oncol. 2013;6:37.

5. Berindan-Neagoe I, Monroig Pdel C, Pasculli B, Calin GA. MicroRNAome genome: a treasure for cancer diagnosis and therapy. CA Cancer J Clin. 2014;64(5):311-36

6. Ponting $\mathrm{CP}$, Oliver PL, Reik W. Evolution and functions of long noncoding RNAs. Cell. 2009;136(4):629-41.

7. Shi $X$, Sun $M$, Liu H, Yao Y, Song Y. Long non-coding RNAs: a new frontier in the study of human diseases. Cancer Lett. 2013;339(2):159-66.

8. Xu Y, Xu Q, Yang L, Ye X, Liu F, Wu F, et al. Identification and validation of a blood-based 18-gene expression signature in colorectal cancer. Clin Cancer Res. 2013;19(11):3039-49.

9. Clemson CM, Hutchinson JN, Sara SA, Ensminger AW, Fox AH, Chess A, et al. An architectural role for a nuclear noncoding RNA: NEAT1 RNA is essential for the structure of paraspeckles. Mol Cell. 2009:33(6):717-26.

10. Sasaki YT, Ideue T, Sano M, Mituyama T, Hirose T. MENepsilon/beta noncoding RNAs are essential for structural integrity of nuclear paraspeckles. Proc Natl Acad Sci U S A. 2009;106(8):2525-30.

11. Mao YS, Sunwoo H, Zhang B, Spector DL. Direct visualization of the co-transcriptional assembly of a nuclear body by noncoding RNAs. Nat Cell Biol. 2011;13(1):95-101.

12. Nakagawa S, Hirose T. Paraspeckle nuclear bodies-useful uselessness? CMLS. 2012;69(18):3027-36.

13. Standaert L, Adriaens C, Radaelli E, Van Keymeulen A, Blanpain C, Hirose T, et al. The long noncoding RNA Neat1 is required for mammary gland development and lactation. RNA. 2014;20(12):1844-9.

14. Nakagawa S, Shimada M, Yanaka K, Mito M, Arai T, Takahashi E, et al. The IncRNA Neat1 is required for corpus luteum formation and the establishment of pregnancy in a subpopulation of mice. Development. 2014;141(23):4618-27.

15. Sunwoo H, Dinger ME, Wilusz JE, Amaral PP, Mattick JS, Spector DL. MEN epsilon/beta nuclear-retained non-coding RNAs are up-regulated upon muscle differentiation and are essential components of paraspeckles. Genome Res. 2009;19(3):347-59.

16. Naganuma T, Hirose T. Paraspeckle formation during the biogenesis of long non-coding RNAs. RNA Biol. 2013;10(3):456-61.

17. Chen W, Zheng R, Zhang S, Zhao P, Li G, Wu L, et al. Report of incidence and mortality in China cancer registries, 2009. Chin J Cancer Res. 2013;25(1):10-21.

18. Siegel R, Ma J, Zou Z, Jemal A. Cancer statistics, 2014. CA Cancer J Clin. 2014;64(1):9-29

19. Levin B, Lieberman DA, McFarland B, Andrews KS, Brooks D, Bond J, et al. Screening and surveillance for the early detection of colorecta cancer and adenomatous polyps, 2008: a joint guideline from the American Cancer Society, the US Multi-Society Task Force on Colorectal Cancer, and the American College of Radiology. Gastroenterology. 2008;134(5):1570-95.

20. Swiderska M, Choromanska B, Dabrowska E, Konarzewska-Duchnowska E, Choromanska K, Szczurko G, et al. The diagnostics of colorectal cancer. Contemp Oncol. 2014;18(1):1-6.

21. Leman ES, Schoen RE, Magheli A, Sokoll LJ, Chan DW, Getzenberg RH. Evaluation of colon cancer-specific antigen 2 as a potential serum marker for colorectal cancer. Clin Cancer Res. 2008;14(5):1349-54.

22. Ran Y, Hu H, Zhou Z, Yu L, Sun L, Pan J, et al. Profiling tumor-associated autoantibodies for the detection of colon cancer. Clin Cancer Res. 2008;14(9):2696-700.

23. Katsila T, Juliachs M, Gregori J, Macarulla T, Villarreal L, Bardelli A, et al. Circulating pEGFR is a candidate response biomarker of cetuximab therapy in colorectal cancer. Clin Cancer Res. 2014;20(24):6346-56.

24. Toiyama Y, Tanaka K, Kitajima T, Shimura T, Kawamura M, Kawamoto A, et al Elevated serum angiopoietin-like protein 2 correlates with the metastatic properties of colorectal cancer: a serum biomarker for early diagnosis and recurrence. Clin Cancer Res. 2014;20(23):6175-86. 
25. Naganuma T, Nakagawa S, Tanigawa A, Sasaki YF, Goshima N, Hirose T. Alternative 3 '-end processing of long noncoding RNA initiates construction of nuclear paraspeckles. EMBO J. 2012;31(20):4020-34

26. Masamha CP, Xia Z, Yang J, Albrecht TR, Li M, Shyu AB, et al. CFIm25 links alternative polyadenylation to glioblastoma tumour suppression. Nature. 2014;510(7505):412-6.

27. Chakravarty D, Sboner A, Nair SS, Giannopoulou E, Li R, Hennig S, et al. The oestrogen receptor alpha-regulated IncRNA NEAT1 is a critical modulator of prostate cancer. Nat Commun. 2014;5:5383.

28. Choudhry H, Albukhari A, Morotti M, Hider S, Moralli D, Smythies J, et al. Tumor hypoxia induces nuclear paraspeckle formation through HIF-2alpha dependent transcriptional activation of NEAT1 leading to cancer cell survival. Oncogene. 2014.

29. Zeng C, Xu Y, Xu L, Yu X, Cheng J, Yang L, et al. Inhibition of long noncoding RNA NEAT1 impairs myeloid differentiation in acute promyelocytic leukemia cells. BMC Cancer. 2014;14:693.

30. West JA, Davis CP, Sunwoo H, Simon MD, Sadreyev Rl, Wang Pl, et al. The long noncoding RNAs NEAT1 and MALAT1 bind active chromatin sites. Mol Cell. 2014;55(5):791-802.

31. Yang MH, Hu ZY, Xu C, Xie LY, Wang XY, Chen SY, et al. MALAT1 promotes colorectal cancer cell proliferation/migration/invasion via PRKA kinase anchor protein 9. Biochim Biophys Acta. 2015;1852(1):166-74.

32. Kao SC, Pavlakis N, Harvie R, Vardy JL, Boyer MJ, van Zandwijk N, et al. High blood neutrophil-to-lymphocyte ratio is an indicator of poor prognosis in malignant mesothelioma patients undergoing systemic therapy. Clin Cancer Res. 2010;16(23):5805-13.

33. Malietzis G, Giacometti M, Askari A, Nachiappan S, Kennedy RH, Faiz OD, et al. A preoperative neutrophil to lymphocyte ratio of 3 predicts disease-free survival after curative elective colorectal cancer surgery. Ann Surg. 2014;260(2):287-92.

34. Tohme S, Sukato D, Chalhoub D, McDonald KA, Zajko A, Amesur N, et al. Neutrophil-lymphocyte ratio is a simple and novel biomarker for prediction of survival after radioembolization for metastatic colorectal cancer. Ann Surg Oncol. 2015;22(5):1701-7.

35. Shen M, Hu P, Donskov F, Wang G, Liu Q, Du J. Tumor-associated neutrophils as a new prognostic factor in cancer: a systematic review and meta-analysis. PLoS One. 2014;9(6), e98259.

36. Wengner AM, Pitchford SC, Furze RC, Rankin SM. The coordinated action of G-CSF and ELR + CXC chemokines in neutrophil mobilization during acute inflammation. Blood. 2008;111(1):42-9.

37. Eash KJ, Greenbaum AM, Gopalan PK, Link DC. CXCR2 and CXCR4 antagonistically regulate neutrophil trafficking from murine bone marrow. J Clin Invest. 2010;120(7):2423-31.

38. Khajah M, Millen B, Cara DC, Waterhouse C, McCafferty DM. Granulocytemacrophage colony-stimulating factor (GM-CSF): a chemoattractive agent for murine leukocytes in vivo. J Leukoc Biol. 2011;89(6):945-53.

39. Reiman JM, Kmieciak M, Manjili MH, Knutson KL. Tumor immunoediting and immunosculpting pathways to cancer progression. Semin Cancer Biol. 2007;17(4):275-87.

40. Himmel ME, Crome SQ, Ivison S, Piccirillo C, Steiner TS, Levings MK. Human CD4+ FOXP3+ regulatory T cells produce CXCL8 and recruit neutrophils. Eur J Immunol. 2011:41(2):306-12.

41. Fridlender ZG, Sun J, Mishalian I, Singhal S, Cheng G, Kapoor V, et al. Transcriptomic analysis comparing tumor-associated neutrophils with granulocytic myeloid-derived suppressor cells and normal neutrophils. PLoS One. 2012;7(2), e31524.

42. Fridlender ZG, Sun J, Kim S, Kapoor V, Cheng G, Ling L, et al. Polarization of tumor-associated neutrophil phenotype by TGF-beta: "N1" versus "N2" TAN. Cancer Cell. 2009;16(3):183-94.

43. Mantovani A, Cassatella MA, Costantini C, Jaillon S. Neutrophils in the activation and regulation of innate and adaptive immunity. Nat Rev Immunol. 2011;11(8):519-31.

44. Fridlender ZG, Albelda SM. Tumor-associated neutrophils: friend or foe? Carcinogenesis. 2012;33(5):949-55.

45. Piccard H, Muschel RJ, Opdenakker G. On the dual roles and polarized phenotypes of neutrophils in tumor development and progression. Crit Rev Oncol Hematol. 2012;82(3):296-309.
46. Imamura K, Imamachi N, Akizuki G, Kumakura M, Kawaguchi A, Nagata K, et al. Long noncoding RNA NEAT1-dependent SFPQ relocation from promoter region to paraspeckle mediates IL8 expression upon immune stimuli. Mol Cell. 2014;53(3):393-406.

47. Zhang Q, Chen CY, Yedavalli VS, Jeang KT. NEAT1 long noncoding RNA and paraspeckle bodies modulate HIV-1 posttranscriptional expression. mBio. 2013;4(1):e00596-12.

48. Imamura K, Akimitsu N. Long Non-Coding RNAs Involved in Immune Responses. Front Immunol. 2014;5:573.

49. Silver N, Best S, Jiang J, Thein SL. Selection of housekeeping genes for gene expression studies in human reticulocytes using real-time PCR. BMC Mol Biol. 2006;7:33.

50. Stamova BS, Apperson M, Walker WL, Tian Y, Xu H, Adamczy P, et al. Identification and validation of suitable endogenous reference genes for gene expression studies in human peripheral blood. BMC Med Genomics. 2009;2:49.

\section{Submit your next manuscript to BioMed Central and take full advantage of:}

- Convenient online submission

- Thorough peer review

- No space constraints or color figure charges

- Immediate publication on acceptance

- Inclusion in PubMed, CAS, Scopus and Google Scholar

- Research which is freely available for redistribution 